\title{
NOMINA NONNULLA NOVA FAMILIAE RUBIACEARUM FLORAE MEXICANAE
}

\author{
A. L. BorHIDI \\ Instituto de Biología, Universidad de Pécs, H-7624 Pécs, Ifjúság útja 6, Hungría \\ E-mail: borhidi@gamma.ttk.pte.hu
}

(Received 11 June, 2018; Accepted 11 July, 2018)

Two species was described as new ones and published in the second edition of the monograph: Rubiáceas de México utilized illegitimate names, which turned to be invalid synonyms (Govaerts 2016). These are rectified in the following paper.

Kew words: Bouvardia, Mexico, new names, Randia, rectification

\section{Bouvardia candida Borhidi, nomen novum}

Nombre reempalzado: Bouvardia candidissima Borhidi, E. Martínez et S. Salas-Morales, Acta Bot. Hung. 53: 68 (2011), Rubiáceas de México, p. 93 (2012), nomen illeg., non Bouvardia candidissima Hovey, Nursery Cat. (Hovey et co.) 1882: 3 (1882).

Tipo: México, Oaxaca, Mpio. Oaxaca de Juárez Jardin Etnobotánico de Oaxaca, $17^{\circ} 03^{\prime} 59^{\prime \prime} \mathrm{N}, 96^{\circ} 43^{\prime} 20^{\prime \prime} \mathrm{W}, 1,545$ m s.n.m. Col.: C. Chávez 21 y E. Martinez, 2 Feb 2001. Holotipo: MEXU.

Bouvardia diversiflora Borhidi et Lozada-Pérez Acta Bot. Hung. 53: 44 (2011).

Tipo: México, Guerrero. Mpio. Atoyac, Loc 16 km al NE de El Paraiso. Alt. 1,100 m s.n.m. Col.: J. C. Soto Núñez (10142) con S. Román G., 19 Aug. 1985. Holotipo: FCME; isotipo: MEXU.

Nombre legitimizado hoc loco. En la descripción original falta la localización de los ejemplares del tipo. Gracias al doctor R. Govaerts por haber llamado nuestra atención a la situación ilegítima anterior de la especie.

Randia simillima Borhidi, nomen novum

Nombre reemplazado: Randia similis Borhidi et S. Salas-Morales, Acta Bot. Hung. 55: 23 (2013), nomen illeg. non Randia similis Craib., Bull. Misc. Inform. Kew, 1911: 391 (1911). 
Tipo: México, Oaxaca, Distr. Tehuantepec, Mpio. Santa María Huamelula. La piedra barca, selva baja caducifolia sobre cerro, suelo pedregoso. Coord.: $16^{\circ} 1^{\prime} 11.6^{\prime \prime} \mathrm{N}, 95^{\circ} 42^{\prime}$ 3.6”'W, Alt.: 80 m s.n.m. Col.: Samuel Molina Bende (SAM) (930), 4 Jan 2011. Holotipo: MEXU; isotipo: SERO.

\section{REFERENCIAS}

Borhidi, A. (2012): Rubiáceas de México. Segunda y ampliada edición. - Akadémiai Kiadó, Budapest, 608 pp.

Borhidi, A., Lozada-Pérez, L. y Salas-Morales, S. (2011): Estudios sobre Rubiáceas Mexicanas. XXXI. Trichosiphon y Gymnosiphon Borhidi, sect nov. Subgen. Bouvardiastrum (Bouvardia Salisb.) con seis especies nuevas. - Acta Bot. Hung. 53: 41-61. https:// doi.org/10.1556/abot.53.2011.1-2.4

Borhidi, A., Martínez-Salas, E. y Salas-Morales, S. (2013): Estudios sobre Rubiáceas Mexicanas XLI. Tres nuevas especies del género Randia L. - Acta Bot. Hung. 55: 17-25. https://doi.org/10.1556/abot.55.2013.1-2.2

Govaerts, R. (2016): World Checklist of Rubiaceae. - Facilitated by the Royal Botanic Gardens, Kew. Published on the Internet; http://wcsp.science.kew.org/ [retrieved 1 June 2018] 